\title{
Correction to: Reactive oxygen species, nitric oxide and plant cell death associated with caspase-like protease activity during somatic embryogenesis in Fraxinus mandshurica
}

Hao Wang ${ }^{1}$ - Dongyan Zhang ${ }^{1}$.

Juan Luis Fernández-Lorenzo ${ }^{2}$ Hailong Shen ${ }^{1}$ •

Ling Yang ${ }^{1}$

Published online: 23 February 2022

(C) Northeast Forestry University 2022

\section{Correction to: J. For. Res.}

https://doi.org/10.1007/s11676-021-01392-y

In this article Ling Yang should have been denoted as a corresponding author. The original article has been corrected.

Publisher's Note Springer Nature remains neutral with regard to jurisdictional claims in published maps and institutional affiliations.

The original article can be found online at https://doi.org/10. 1007/s11676-021-01392-y.

Ling Yang

yangl-cf@nefu.edu.cn

Hailong Shen

shenhl-cf@nefu.edu.cn

1 State Key Laboratory of Tree Genetics and Breeding, School of Forestry, Northeast Forestry University, Harbin 150040, People's Republic of China

2 Crop Production Department, High Polytechnique School of Engineering, Santiago de Compostela University, Lugo, Spain 